\title{
PERCEPCION POR PARTE DE LOS PROFESORES DE EDUCACIÓN FÍSICA SOBRE DOCENCIA EFICAZ, EN ESTABLECIMIENTOS MUNICIPALES CON ALTOS ÍNDICES DE VULNERABILIDAD ESCOLAR DE LA CUIDAD DE VALPARAÍSO
}

\section{PHYSICAL EDUCATION TEACHERS OPINIONS ON EFFECTIVE TEACHING IN PUBLIC SCHOOLS WITH HIGH LEVEL OF SOCIAL VULNERABILITY IN VALPARAISO CITY}

\author{
Cajas Luna, Boris; León León, Mario; Mancilla Guzmán, Marcelo; Osorio Gutiérrez, Jesús.
}

GICEFYD, Grupo de Investigación en Ciencias de Ejercicio Físico y del Deporte, Carrera Pedagogía en Educación Física, Universidad Viña Del Mar.

CAJAS L.B.; LEON L.M.; MANCILLA G.M.; OSORIO G.J. (2013). Percepción por parte de los profesores de educación física sobre docencia eficaz, en establecimientos municipales con altos índices de vulnerabilidad escolar de la ciudad de Valparaíso. Mot. Hum. 14(1): 16-21.

\section{RESUMEN}

Esta investigación de tipo caso descriptiva pretende conocer las percepciones por parte de los profesores de educación física sobre docencia eficaz, en establecimientos municipales con altos índices de vulnerabilidad escolar de la cuidad de Valparaíso. Se llevó a cabo en el período Agosto- Noviembre del año 2012. Para ello se utilizaron técnicas cualitativas; entrevista en profundidad no estructurada con preguntas abiertas. Expone contenidos relacionados con vulnerabilidad escolar, establecimientos municipales, docencia eficaz y las implicancias, de ésta, según estudios en el desempeño del docente y también en el proceso de enseñanza-aprendizaje. Se espera que esta investigación, otorgue información básica y detallada que permita fundamentar estudios posteriores de mayor profundidad y análisis, como también ser la base de futuros proyectos de acción en el campo del contexto educacional específicamente en el área de la docencia y el perfeccionamiento del mismo.

Palabras clave: Docencia eficaz, Vulnerabilidad, Establecimientos municipales.

ABSTRACT
This research presents a type of descriptive case that pretends to know the perceptions of the physical education teachers
about effective teaching in municipal establishments with high levels of school vulnerability in the city of Valparaiso. This
was conducted during the period of August- November of the year 2012. For this there were used many qualitative
techniques such as; detailed interviews not structured with this we refer to opened question for physical education
teachers, to know the perceptions about effective teaching by physical education teachers. It exposes contents related
with school vulnerability, municipal establishments, effective teaching and the implications of it, through the studies done
in the performance of the teachers and also in the process of teaching-learning. We expect that this research will give
basic information and detailed that permits to develop post studies of mayor depth and analysis, like also be a basis for
future projects of action in the campus of specifically educational context in the area of teaching and improvement of the
same.
wey
words:
effective




\section{INTRODUCCIÓN}

Desde la década del 90 una de las líneas de investigación educativa que más está influenciando en la toma de decisiones para mejorar la calidad de la educación en todo el mundo, incluyendo Latinoamérica, es el movimiento teórico-practico de Docencia eficaz escolar. Sin embargo, esa influencia no siempre es clara ni directa, en ocasiones es utilizada de forma parcial, interesada y sesgada, con lo que sus efectos pueden resultar incluso contraproducentes (Murillo, 2003).

Con el pasar de los años, las circunstancias en que las sociedades deben demostrar su nivel de competencia se van haciendo más comunes, esto ha llevado a la búsqueda de un término adecuado para describir dicha idoneidad, esperando así lograr establecer cuál es la colectividad que se encuentra en condiciones óptimas de desarrollo a nivel global. Siguiendo esta dinámica, es que se ha intentado definir de una manera simple esta característica y es así como se ha llegado al término de eficacia o eficiencia.

Por otra parte, en el proceso de equidad que el gobierno plantea para la sociedad chilena, el sector educación aparece como una de las áreas prioritarias a desarrollar. Para lograrlo, se plantea los siguientes objetivos: Ampliar la cobertura educacional y mejorar la calidad de la educación, impulsando prácticas pedagógicas innovadoras para promover aprendizajes activos y pertinentes a los diferentes contextos socioculturales de los alumnos, otorgando más oportunidades de perfeccionamiento a los docentes y aumentado la inversión en el área con recursos públicos y privados, entre otros. (MIDEPLAN, 2011).

$\mathrm{Si}$ bien todas estas iniciativas revisten gran importancia, resultan insuficientes para mejorar realmente la educación, ya que la preocupación de la autoridad no ha recaído como debiera sobre el recurso humano, fundamentalmente para llegar a concretar los objetivos anteriormente señalados. Es el profesor, quien percibe cómo los acelerados cambios sociales influyen negativamente en su personalidad, produciéndole un gran malestar al momento de ejercer su labor docente (Esteve \& Rajecevic, 1998).

La calidad de los profesores, a su vez, va en función de la maestria que éste logre sobre las materias, el conocimiento que tenga y el uso que haga de las técnicas pedagógicas adecuadas y el tener actitudes profesionales adecuadas (Ávalos \& Haddad, 1981).

En Chile investigaciones demuestran que el profesor es un agente que tiene incidencia en el aprendizaje escolar de los niños. Los resultados de estas investigaciones entregan evidencia que hay un cambio en el rendimiento académico en alumnos de ciertos profesores, independientemente de los antecedentes familiares del niño y las características de la institución escolar. Por lo tanto, concluyen que existe un campo de estudio específico en relación a las características personales, actitudinales y su relación con el aprendizaje de los alumnos (Arancibia \& Álvarez, 1991).

Los resultados de estas investigaciones demuestran empíricamente el supuesto sobre la importancia del profesor en el aprendizaje del niño en el sistema escolar actual, por lo tanto intervenir a nivel de los profesores, parece ser la manera más directa de mejorar la calidad de la educación. De esto podemos inferir que el profesor es quien debe estar presente y ejercer un protagonismo en el proceso de cambio de la educación, dado su impacto en ésta.

Aptitudes como la habilidad para realizar actividades de carácter práctico, entender las emociones de sus alumnos y discriminar cuáles son positivas o negativas y como éstas influirán en el proceso de enseñanza-aprendizaje, son características que otorgarán el necesario grado para ser considerado como competente.

La pedagogía como ciencia, debe ir considerando sobre la marcha, diferentes factores que puedan beneficiar los modelos de enseñanza en los diversos establecimientos, uno de éstos es la Docencia Eficaz, concepto estrechamente ligado a la inteligencia emocional; y como educación, un tipo de inteligencia social, que un educando en el área de la Educación Física debe incluir dentro de sus cualidades y capacidades para ser catalogado como un pedagogo competente. 
CAJAS L.B.; LEON L.M.; MANCILLA G.M.; OSORIO G.J. (2013). Percepción por parte de los profesores de educación física sobre docencia eficaz, en establecimientos municipales con altos índices de vulnerabilidad escolar de la ciudad de Valparaíso. Mot. Hum. 14(1): 16-21.

Considerando la Docencia Eficaz como un componente relevante en la educación, esta investigación indaga cómo factores directos e indirectos tanto personales, sociales, emocionales y afectivos, pueden influir en el rol del docente dentro de la comunidad escolar, pasando por situaciones tan cotidianas de su labor educativa, como el nivel de comodidad que éste pueda presentar en su lugar de trabajo, hasta qué tipo de emociones siente el pedagogo cuando está frente a un grupo de alumnos y de qué manera estas influyen en la interacción con éstos.

Se establece, que un profesor con una percepción o sentido débil de docencia eficaz, centrara su clase en reglas estrictas, castigos y a su vez, ven con pesimismo la motivación de los educandos para con los contenidos curriculares, en absoluto contraste a un docente con sentido elevado de eficacia, que es capaz de establecer nuevos métodos y estrategias, desarrollar nuevas capacidades lo que genera influencia directa sobre el proceso enseñanzaaprendizaje y a su vez sobre la educación.

\section{MATERIAL Y METODOS}

Corresponde a un estudio cualitativo, de tipo casodescriptivo, de caso porque es un método que permite detallar situaciones, efemérides, sucesos. Lo que quiere decir es cómo se manifiestan o cómo son determinados fenómenos en nuestra sociedad. Descriptivo en tanto "los estudios descriptivos buscan especificar las propiedades, las características y los perfiles importantes de las personas, grupos y comunidades o cualquier otro fenómeno que se somete a un análisis" (Fernández, 2003).

Esencialmente esta investigación busca comprender e interpretar la realidad tal y cómo es entendida por los sujetos participantes en los contextos estudiados, ya que este método de investigación, acerca de una situación compleja, se basa en el entendimiento comprensivo de dicho escenario, el cual se obtiene a través de la descripción y análisis de la situación, la cual es tomada como un conjunto y dentro de un contexto (Gómez \& Gonzáles, 2011).

Este estudio contempla los atributos; Docencia Eficaz, Establecimiento, Profesor de Educación Física, Vulnerabilidad. De los atributos mencionados, y luego del análisis de discurso, surgen las categorías que llevan el mismo nombre que los atributos debido al perfecto encaje semántico.

Respecto a la población utilizada para este estudio, estuvo compuesta por 8 profesores de educación física de establecimientos municipales con altos índices de Vulnerabilidad Escolar de la Ciudad de Valparaíso.

\section{Criterios de Inclusión}

Para los profesores: Profesores titulados de Educación Física, con 7 años de experiencia y 4 años dentro del establecimiento.

Para los establecimientos: Establecimientos municipales vulnerables que pertenezcan a la Corporación Municipal de Valparaíso.

\section{Criterios de exclusión}

Para los Profesores: que sean profesores egresados no titulados, profesores de reemplazo y/o practicante. Para los Establecimientos: Establecimientos que no pertenezcan a la Corporación Municipal de Valparaíso y que no sean establecimientos municipales ni vulnerables.

La muestra es no probabilística e intencionada. La técnica utilizada para la recolección de datos fueron entrevistas en profundidad, entendiendo la entrevista en profundidad como una "herramienta de excavar" favorita de los sociólogos. Para adquirir conocimientos sobre la vida social, los científicos sociales reposan en gran medida sobre relatos verbales. Estos conocimientos verbales servirán para adquirir todo tipo de información que pueda servir a la investigación, junto con la obtención de datos para compilar la mayor cantidad de datos relevantes, que contribuyan a la investigación (Bernney \& Hughes, 2008).

\section{RESULTADOS}

En el discurso de los docentes se expresa la percepción acerca de Docencia Eficaz, lo que a su vez demuestra estar alejada de la teoría pero también se expresa la clara influencia de las emociones sobre el accionar docente para generar un aprendizaje 
CAJAS L.B.; LEON L.M.; MANCILLA G.M.; OSORIO G.J. (2013). Percepción por parte de los profesores de educación física sobre docencia eficaz, en establecimientos municipales con altos índices de vulnerabilidad escolar de la ciudad de Valparaíso. Mot. Hum. 14(1): 16-21.

significativo dentro de los alumnos pertenecientes a los establecimientos vulnerables de Valparaíso abarcados por este estudio.

En base a los discursos de los profesores y la teoría se aprecia que en la enseñanza, influye directamente la formación del educador en su área específica, como también a su vez el desarrollo y el manejo de los contenidos, saber abordarlos de acuerdo a las cualidades del curso que se le haya destinado, demostrando conseguir logros en aprendizajes en diferentes grupos etáreos, independiente de su cualidad económica, social o afectiva.

Por otra parte, las condicionantes que influyen en una docencia eficaz, se encuentran las características psicológicas del profesor en el contexto educativo. Por tal motivo considerando la teoría, quien imparta la docencia en ambientes con altos índices de vulnerabilidad estudiantil, deberá poseer características psicológicas idóneas para lograr una real docencia eficaz. En el caso de que estas cualidades psicológicas no se cumplan, se deberán trabajar en ellas, para que el educador que vaya a instruir a los alumnos más vulnerables, sea la persona más idónea, aplicando la tolerancia, la paciencia, la honestidad, concentrarse en lo valórico-afectivo y por sobre todo poseer un dominio del temperamento.

Se aprecia además la importancia y el respeto que entregue el profesor de educación física y el establecimiento educacional a la clase de educación física para concretar los objetivos que ésta requiere, desde la formación académica, moral y corporal para el desarrollo óptimo del alumno. De tal manera que depende del profesor el buen uso que le dé a su clase y cómo optimiza los tiempos necesarios para lograr los objetivos de ésta. No obstante, se evidenció que los establecimientos educacionales en ocasiones, ocupan las horas de educación física para otras actividades, ocupan el patio (que es su sala de clases) y es la asignatura de educación física la que debe estar disponible para la realización de otras actividades, modificándose los horarios originales de éstas, e incluso no recuperándolas.

Se destaca también, en el discurso de los docentes, que el desarrollo de una Docencia Eficaz en este tipo de establecimiento se torna más complejo al considerar que éstos y los educandos se ven afectados por diferentes factores de índole negativo que impiden el normal desarrollo de un proceso educativo acorde a lo establecido por las bases curriculares, dentro de estos factores se mencionan; problemas económicos, de alimentación, problemas de vestimenta, familias disfuncionales, ambientes de agresividad, poco compromiso del núcleo familiar con la educación del alumno, los cuales claramente influenciaran la escolaridad de cada uno de los alumnos.

Señalan también, (los profesores en su discurso) que es en el establecimiento educacional, donde los alumnos pasarán la mayor parte de su tiempo, relacionándose con: personal administrativo, docentes y compañeros de curso. Pero de manera más importante, es con estos dos últimos con quienes desarrollará mayores relaciones interpersonales, esto tiene especial influencia sobre el bienestar general del alumno, entendiendo que, estas relaciones interpersonales estén basadas en el respeto mutuo, cariño y compresión. En el contexto afectivo, el alumno podrá mantener una base de confianza y apertura a nuevos conocimientos, ayudado por el respaldo y aprecio de sus pares y lo variable de sus actitudes u opiniones, pudiendo fomentar de mejor manera aspectos volitivos de parte de los pedagogos hacia los estudiantes.

También, de existir una infraestructura (en éstos establecimientos clasificados como vulnerables) orientada al deporte y la realización efectiva de las clases de Educación Física, donde no se pasara a llevar la labor docente, se esperaría que éstas fuesen mucho más compenetradas con las necesidades de los alumnos y orientadas desarrollo integral en cada una de sus clases prácticas.

Los docentes, manifiestan que resulta difícil establecer una Docencia Eficaz en ambientes vulnerables, puesto que ésta deja de lado factores relevantes que podrían modificar, influenciar y determinar logros educativos tanto de profesores como de alumnos, todo esto determinado por las condiciones del entorno que rodean al alumno, más aún, si actualmente en nuestro país, los logros educativos están medidos principalmente por el rendimiento académico que logre alcanzar un alumno, dejando de lado otras capacidades y valores 
que pueden aprender o desarrollar en comunidad, como respeto, compañerismo, trabajo en equipo, entre otros. Esto último, para un nivel educativocompetitivo, como el actual, no es preponderante al momento de evaluar a estos alumnos.

\section{CONCLUSION}

La docencia eficaz esta asociada a una personalidad determinada y definida por parte de los docentes. El profesor debe ser un ente socializador, no solo en lo que respecta a la entrega de contenidos teóricos de formación, sino ser capaz de vincularse a través de valores en la formación integral del alumno, crear lazos con sus pares en función de un accionar pedagógico transversal, que apunte a objetivos comunes de formación de los educandos, evitando ambientes laborales desagradables, enfocándose a una enseñanza más cercana y directa, transformando la comunidad escolar en un contexto con características de familia.

Los profesores tienen una percepción sesgada de docencia eficaz, las cual no responde ni concuerda con los constructos teóricos de ésta, menos en su práctica pedagógica, mas bien la señalan en función de características personales basadas en sus experiencias laborales. Ser un docente eficaz, involucra realizar una docencia que tenga como eje central los valores, como también, plantear objetivos acordes al contexto en el cual se desempeñan.

Existen factores Bio-Psico-Sociales de la comunidad y el contexto escolar, que dificultan el accionar pedagógico, sumado a las características de los alumnos (carencias afectivas, valóricas y actitudinales). Es poco viable llevar a cabo una docencia eficaz en establecimientos vulnerables, debido a que la infraestructura física y de recursos materiales no es la adecuada para desarrollar metodologías de enseñanza-aprendizaje de forma óptima.

Los profesores que desarrollen su labor docente en establecimientos con altos índices de vulnerabilidad, deben poseer capacitación, propia del trabajo en estos ambientes, demostrar un dominio de la teoría, entendiendo esto desde la didáctica y las estrategias pedagógicas innovadoras que motiven a los alumnos. El proceso evaluativo no debe centrarse solamente en el resultados, ya que estudios demuestran que en contextos educativos vulnerables, este factor no es preponderante al momento de establecer avances en un proceso educativo.

A nivel país, se requiere establecer políticas educacionales dirigidas a contextos vulnerables, que fomenten y sustenten, la permanencia y desarrollo de cada uno de los establecimientos, y que a su vez proporcionen los recursos necesarios para fortalecer los procesos de enseñanza-aprendizaje de los alumnos.

Finalmente, aspectos de la teoría que no son considerados como vulnerables o que influyen en una docencia eficaz son los denominados aspectos complejos; como problemas económicos, de alimentación, de vestimenta, familias monoparentales, agresividad, poco apoyo de familiares significativos en el proceso de educación de éstos alumnos, aspectos a considerar para el logro de objetivos educacionales dentro de un contexto de vulnerabilidad escolar.

\section{REFERENCIAS BIBLIOGRAFICAS}

Arancibia, V. y Álvarez, M.I. (1991). Modelo de variables del profesor y su impacto en rendimiento escolar y autoconcepto académico. Proyecto Fondecyt $\mathrm{N}^{\circ} 1880405$. Santiago de Chile: Pontificia Universidad Católica de Chile.

Ávalos, B \& Haddad, W (1981). Reseña de la Investigación sobre Efectividad de los maestros en África, America Latina, Filipinas, India, Malasia, Medio Oriente y Tailandia: Resultados. Ottawa: CIID.

Bernney y Hughes. (2008). Métodos cualitativos aplicados 2.

Esteve, A. Rajecevic, B. (1998). El profesor como investigador en el aula: un paradigma de formación de profesores. Madrid.

Fernández, B. (2003). Métodos y Técnicas cualitativas de investigación en ciencias sociales. Madrid.

Gómez, M y Gonzáles, P. (2011). Metodología cualitativa en ciencias sociales y educación. Orientaciones teórico metodológicas y técnicas de investigación. Chile.

Ministerio de Planificación Nacional (2011). Información General de las Metas de las Acciones Estratégicas del PND 2011-2014, Sector Educación. Chile. 
CAJAS L.B.; LEON L.M.; MANCILLA G.M.; OSORIO G.J. (2013). Percepción por parte de los profesores de educación física sobre docencia eficaz, en establecimientos municipales con altos índices de vulnerabilidad escolar de la ciudad de Valparaíso. Mot. Hum. 14(1): 16-21.

Murillo, F (2003). La investigación sobre eficacia escolar en Iberoamérica. Revisión internacional del estado de la cuestión. Bogota.

\section{RECIBIDO: 28-12-2012}

ACEPTADO: 31-03-2013

\section{Dirigir Correspondencia a:}

Boris Cajas Luna

Agua Santa 7055, Rodelillo, Viña del Mar, Chile.

Email: bcajas@gmail.com 